\title{
Developing a handheld record for patients with cystic fibrosis
}

This article was published in the following Dove Press journal:

Patient Related Outcome Measures

13 August 2015

Number of times this article has been viewed

\author{
Omendra Narayan' \\ Siobhan Davies' \\ Carly Tibbins ${ }^{2}$ \\ JH Martyn Rees ${ }^{3}$ \\ Warren Lenney',4 \\ Francis J Gilchrist ${ }^{1,4}$ \\ 'Academic Department of Child \\ Health, Royal Stoke University \\ Hospital, Stoke-on-Trent, ${ }^{2}$ West \\ Midlands Medicines for Children \\ Research Network, Royal Stoke \\ University Hospital, Stoke-on-Trent, \\ ${ }^{3}$ Department of Paediatrics, Royal \\ Shrewsbury Hospital, Shrewsbury, \\ ${ }^{4}$ Institute for Science and Technology \\ in Medicine, Keele University, Guy \\ Hilton Research Centre, Stoke-on- \\ Trent, UK
}

\begin{abstract}
Patient handheld records (PHHRs) promote self-management and empower the holder to take a more active role in the management of their disease. They have been used successfully in improving preventative care for children and have contributed to improved adherence in a number of chronic illnesses. Despite the potential advantages, there are no standard PHHRs for patients with cystic fibrosis (CF). We report the consultation process that led to the development of a CF PHHR, describe the final document, and analyze the feedback from their use at our center. We have made the CF PHHR freely available online.
\end{abstract}

Keywords: cystic fibrosis, pediatrics, patient and public involvement, patient handheld records

\section{Introduction}

Effective self-management starts with the education of the patient to improve understanding of their medical condition. They are then encouraged to monitor the aspects of their illness and eventually to tailor their treatment accordingly. This empowers the patients to take control of their illness and helps optimize their care. ${ }^{1}$ Patient handheld records (PHHRs) are one way to promote self-management and have been successful particularly with regard to infant immunizations. ${ }^{2,3}$ They have also been shown to improve adherence to treatment in patients with chronic conditions such as inflammatory bowel disease and diabetes. ${ }^{46}$ PHHRs help teach patients about their specific disease and improve the understanding of treatments, thereby encouraging active participation in self-management. ${ }^{6}$ The Department of Health, England, supports self-management programs. It has established the Expert Patient Program and published The expert patient: a new approach to chronic disease management for the twenty-first century. ${ }^{7}$

Cystic fibrosis (CF) is a life-limiting disease characterized by cycles of respiratory infection, chronic inflammation, and lung damage. There are also many complications that affect virtually all systems of the body. Even when well, children with $\mathrm{CF}$ are usually on multiple medications and therapies. ${ }^{8}$ They are reviewed regularly in the outpatient clinic, and fundamental to their care is the multidisciplinary team. The CF multidisciplinary team tries to ensure that children receive the best quality care, but this can lead to children and their parents being given large amounts of information from multiple professionals at a single outpatient visit. As patients and parents only retain a small fraction of the information given verbally, it is important that additional steps are taken to improve how much information is retained. ${ }^{9}$ Parents are regularly asked
Correspondence: Francis J Gilchrist Academic Department of Child Health, Royal Stoke University Hospital, Newcastle Road, Stoke-on-Trent ST4 6QG, UK

Tel +44 I78 2675289

Fax +448436365 389

Email francis.gilchrist@uhns.nhs.uk 
about previous medicines, such as the names, doses, dates of previous courses, and presence of allergic reactions or side effects. Such facts are difficult to recall in detail. Increasing the patient's and parent's understanding of the disease and its treatments is vital in improving adherence with medication and therapies. This is associated with better outcomes.

There is no standardized PHHR for CF patients in the UK. Following the successful introduction of PHHR for children with asthma in our center, we decided to introduce them for children with CF. The aim of the CF PHHR was to provide a single point of entry for all information and data relevant to the child. Ultimately, it was hoped that this would empower the parent and child to feel more in control of their disease. We wanted clinical decisions to continue to be made solely by the physician but informed by more accurate information from the patient. Close and early contact with families continued to be encouraged.

\section{Consultation process}

Taking part in the consultation process was entirely voluntary and the families all volunteered to take part. Verbal consent was taken by the User Involvement Coordinator prior to the interviews commencing. The authors advise that according to the NHS National Research Ethics Service guidance, ethical approval was not required as this was a service evaluation project. We undertook an extensive consultation process involving 55 families in which there was at least one child with CF. Interviews were undertaken with the parents/ guardians and, where appropriate, the child together with family members. The interviews were facilitated by a User Involvement Coordinator from the West Midlands Medicines for Children Research Network who was not part of the CF team. The West Midlands was one of the first regions to employ User Involvement Coordinators, and they have extensive experience in working with children and families. The interviews were carried out in the outpatient's clinic at the end of a routine appointment to avoid the inconvenience of additional visits. The interviews were structured to gain opinions on the need for a CF PHHR and ideas for what should be included. Discussions were recorded and transcribed. The transcripts were then analyzed to identify emerging themes. In a separate process, an Expert Patient Advisor from the UK CF Trust used a patient forum to invite comment from patients and families about the value of developing a CF PHHR.

\section{Results of the consultation process}

There was a universal positivity toward the development of CF PHHR among parents and children. It was thought that it would be most useful for the parents whose children had recently been diagnosed with $\mathrm{CF}$, as those who had lived with the disease for a number of years had developed their own systems for recording and storing information. Some of the teenage patients stated that their parents had taken the responsibility for their CF all their lives, so they would not be bothered or remember to use PHHR. Other teenagers said that if they had grown up with PHHR it might encourage them to take more responsibility at an earlier age. One young person stated "I don't like to think about my condition when I'm well so I would only use it if I was ill, then it might be helpful". The format of the CF PHHR was discussed at length. Parents generally were in favor of a paper format for ease of entering and storing data. Some teenagers were more in favor of an electronic version, although they all stated that they would still be likely to forget to complete it.

\section{Important themes highlighted by the consultation process}

PHHR must be given to families when the diagnosis is first made to help increase understanding in all family members.

- It would be a useful tool to take on holidays and school outings.

- The child should be allowed to individualize the front cover.

- It should be possible to alter the content according to the particular requirement of individual families. This will allow the PHHR to evolve as the child gets older and the severity of the disease changes.

- Older children/teenagers might wish to use it to express feelings or anxieties. This would encourage such issues to be shared with health care professionals without needing to be questioned directly, especially on topics that were embarrassing or difficult to articulate.

\section{Specification of the CF PHHR}

The children and parents identified the best hard copy form for the CF PHHR was as an A5 ring binder file. This included a removable front cover to allow the child to personalize it. The file has eight sections, but patients/parents can remove sections not relevant to them. The sections are:

- Front sheets

- Patient's name

- If found, details of where to return file to

- Details of CF team

- Contact telephone numbers 
- Section 1 - Information sharing

- Day-to-day diary to record issues and comments

- Section to record information to and from CF team

- Section to record dates and times of appointments

- Section 2 - Medications

- Table to record current and previous medications

- Table to record drug allergies/intolerances

- Tables to record details of oral and intravenous antibiotic course

- Treatment and prophylaxis oral antibiotic guidelines

- Table to record dates of port flushes

- Nebulized treatments diary

- Table to record vaccinations

- Section 3 - Ages and stages

- Guide for families to check that their child's understanding of CF is age appropriate

- When appropriate the transition documentation will be added to this section

- Section 4 - Investigations

- Brief information about relevant investigations

- Personal plan with chart to record dates of all investigations

- Section 5-Growth and nutrition

- Table and growth chart to record height and weight

- Food, calorie, and enzyme information

- Table to record current enzyme dose

- CF related diabetes information will be added to this section is appropriate

- Section 6-Respiratory

- Table and graph to plot lung function results over time

- Table to record details of equipment on loan

- Section 7 - General information

- Appropriate information can be included regarding: holidays, travel insurance, schools, university, housing, benefits, and support groups

- Section 8-Glossary

- Glossary of common medical terms used in CF.

\section{Implementation of CF PHHR}

The prototype CF PHHR was piloted in seven families with different backgrounds and demographics. After a 4-month trial period, direct feedback resulted in some minor amendments. The CF PHHR was then printed and distributed to all families attending the North West Midlands CF Center.

\section{Assessment of CF PHHR}

One year after the introduction of the CF PHHR, patients and families were surveyed to assess its usefulness. This was done using an anonymized 15 item questionnaire. The questionnaire was completed by the older children themselves and the parents of younger children. A total of 32 families completed the survey. In all, 24/32 (75\%) found it useful or extremely useful and 24/32 used it regularly at their clinic appointments. Parents whose children were newly diagnosed children with CF were more likely to use it. In all, 11/32 (33\%) used it when on holiday as a place to record important information in case of emergencies. Comments included: "it is a Godsend" and "this is my memory!" We would like to formally evaluate the CF PHHR in a more scientific way. We are planning to develop a further questionnaire to discover which specific features within the CF PHHR have been most helpful to the children and their families and which would benefit from being modified. In the future we also hope to assess if the use of CF PHHR is associated with better clinical outcomes (lung function and body mass index) and a better understanding of CF when this assessed as the patient transitions from pediatric to adult care.

\section{Conclusion}

PHHR is an inexpensive and practical tool that can increase and improve patient and parent knowledge of a specific disease such as CF. This can also encourage patients to manage their own symptoms and get involved in their care, which can have a positive effect on their overall health. The development of PHHR specific to CF has been well received by children and parents alike. We believe that the key to this success was the extensive consultation process that guided their development and the ease with which they can be modified to suit individual needs. The CF PHHR provides a single place to record data, store documents, and $\log$ thoughts or questions. We believe that it will encourage children and parents to take a more active role in the child's illness. Families find this especially useful when going away on holidays or during health care appointments. Our latest version of the CF PHHR is available via the journals webpage free of charge. The design of the CF PHHR is under constant review and informed by feedback from patients and their families. It would be relatively easy to develop a version of the CF PHHR as a smart phone app. This is likely to improve usability and access as individuals are rarely without their phones. This idea has been met with interest and enthusiasm especially by the young patients and we are looking to take this forward in the near future.

\section{Disclosure}

The authors report no conflicts of interest in this work. 


\section{References}

1. Schreurs KM, Colland VT, Kuijer RG, de Ridder DT, van Elderen T. Development, content, and process evaluation of a short self-management intervention in patients with chronic diseases requiring self-care behaviours. Patient Educ Couns. 2003;51(2):133-141.

2. Dickey LL. Promoting preventive care with patient-held minirecords: a review. Patient Educ Couns. 1993;20(1):37-47.

3. McCormick MC, Shapiro S, Starfield BH. The association of patient-held records and completion of immunizations. Clin Pediatr (Phila). 1981;20(4):270-274.

4. Saibil F, Lai E, Hayward A, Yip J, Gilbert C. Self-management for people with inflammatory bowel disease. Can J Gastroenterol. 2008; 22(3):281-287.
5. Rubin RR. Adherence to pharmacologic therapy in patients with type 2 diabetes mellitus. Am J Med. 2005;118(Suppl 5A):27S-34S.

6. Turner KE, Fuller S. Patient-held maternal and/or child health records: meeting the information needs of patients and healthcare providers in developing countries? Online J Public Health Inform. 2011;3(2). Available from: http://www.ncbi.nlm.nih.gov/pmc/articles/PMC3615781/.

7. Tattersall RL. The expert patient: a new approach to chronic disease management for the twenty-first century. Clin Med. 2002;2(3):227-229.

8. Ziaian T, Sawyer MG, Reynolds KE, et al. Treatment burden and health-related quality of life of children with diabetes, cystic fibrosis and asthma. J Paediatr Child Health. 2006;42(10):596-600.

9. Kessels RP. Patients' memory for medical information. J R Soc Med. 2003;96(5):219-222.

\section{Publish your work in this journal}

Patient Related Outcome Measures is an international, peer-reviewed, open access journal focusing on treatment outcomes specifically relevant to patients. All aspects of patient care are addressed within the journal and practitioners from all disciplines are invited to submit their work as well as healthcare researchers and patient support groups.
The manuscript management system is completely online and includes a very quick and fair peer-review system. Visit http://www.dovepress. com/testimonials.php to read real quotes from published authors.

\footnotetext{
Submit your manuscript here: http://www.dovepress.com/patient-related-outcome-measures-journal
} 